\title{
O Impacto do Implante Coclear na Linguagem Oral das Crianças com Surdez Congénita
}

\author{
The Impact of Cochlear Implant in the Oral Language of Children with \\ Congenital Deafness
}

\author{
Daniela RAMOS ${ }^{1}$, João XAVIER JORGE ${ }^{2}$, António TEIXEIRA ${ }^{3}$, Carlos RIBEIRO ${ }^{1}$, António PAIVA ${ }^{1}$ \\ Acta Med Port 2015 Jul-Aug;28(4):442-447
}

\section{RESUMO}

Introdução: As crianças com surdez neurossensorial severa a profunda podem adquirir vocabulário e estruturas sintácticas para comunicarem pela linguagem oral, após implante coclear.

Objectivo: Identificar as capacidades linguísticas em crianças, com implante coclear.

Material e Métodos: Estudou-se a linguagem oral em 18 crianças, entre nove e 10 anos, com surdez neurossensorial profunda bilateral congénita, com implante coclear, avaliadas com a Grelha de Observação da Linguagem-Nível Escolar. As pontuações obtidas foram comparadas com as das crianças normo-ouvintes com igual idade auditiva.

Resultados: As pontuações nas estruturas linguísticas estudadas, crianças implantadas vs. padrão das normo-ouvintes foram: fonologia 29,44 $\pm 8,4$ vs. $29,68 \pm 5,90, p=0,91$; semântica 18,55 $\pm 8,89$ vs. $19,20 \pm 4,85, p=0,76$; morfossintaxe $21,89 \pm 12,85$ vs. $26,35 \pm$ $10,36, p=0,159$. Nas provas da estrutura semântica, não se registaram diferenças estatisticamente significativas. Na morfossintaxe, a diferença foi estatisticamente significativa na derivação das palavras, $2,83 \pm 2,81$ vs. $4,65 \pm 1,64, p=0,014$. Na fonologia, verificou-se diferença significativa, na prova de discriminação de pseudopalavras, $6,6 \pm 2,8$ vs. $8,37 \pm 2,32, p=0,023$. Na segmentação silábica, as crianças implantadas tiveram uma pontuação significativamente superior ao padrão 8,56 $\pm 1,6$ vs. 5,9 $\pm 1,58, p<0,001$.

Discussão: A semelhança das pontuações obtidas pelas crianças com implante coclear em relação ao padrão nas componentes linguísticas estudadas, confirma que o implante coclear promove o desenvolvimento da linguagem verbal oral nas crianças com surdez congénita.

Conclusão: As crianças implantadas obtiveram ganhos de linguagem similares às normo-ouvintes com igual idade auditiva.

Palavras-chave: Acústica da Fala; Criança; Desenvolvimento da Linguagem; Implantes Coclears; Percepção da Fala; Perda Auditiva Neurossensorial.

\section{ABSTRACT}

Introduction: Children with severe to profound sensorineural deafness can acquire vocabulary and syntactic structures to communicate by oral language, after cochlear implant.

Aim: Identify the linguistic skills of children with cochlear implant.

Material and Methods: Eighteen children of both gender, between 9 and 10 years, with congenital bilateral deafness, using cochlear implant, were studied. The evaluation instrument used was Observation Chart of Language-School Level. The results were compared with standard of normal-hearing children with the same hearing age.

Results: The scores registered in the linguistics structures studied, comparing implanted children and standard, was: phonology, 29.44 \pm 8.4 vs. $29.68 \pm 5.90, p=0.91$; semantics, $18.55 \pm 8.89$ vs. $19.20 \pm 4.85, p=0.76$; morpho-syntax $21.89 \pm 12.85$ vs. $26.35 \pm 10.36$, $p=0.159$. Regarding the tests of semantics, there was no significant difference. Concerning the tests of morpho-syntactic structure, the difference was significant in the derivation of words, $2.83 \pm 2.81$ vs. $4.65 \pm 1.64, p=0.014$. In the phonology, a significant difference was found comparing implanted children and standard, in the discrimination of pseudo words, $6.6 \pm 2.8 \mathrm{vs}$. $8.37 \pm 2.32, p=0.023$. However, in syllabic segmentation, implanted children had a mean score $8.56 \pm 1.6$ significantly higher than standard, $5.9 \pm 1.58, p<0.001$.

Discussion: The similarity of the scores obtained by children with cochlear implants with the standard, in the language components studied confirms that cochlear implant promotes the development of oral verbal language in children with congenital deafness.

Conclusions: Implanted children had acquired language skills similar to normal-hearing children with the same hearing age.

Keywords: Child; Cochlear Implants; Hearing Loss, Sensorineural; Language Development; Speech Acoustics; Speech Perception.

\section{INTRODUÇÃO}

A surdez neurossensorial severa a profunda apresenta grande impacto na aquisição e desenvolvimento da linguagem verbal oral, comprometendo o desempenho linguístico e escolar das crianças portadoras deste tipo e grau(s) de surdez. ${ }^{1}$

O implante coclear é um elemento facilitador do desenvolvimento da linguagem em crianças com surdez neurossensorial severa a profunda congénita, nas quais é limitado o benefício da correcção auditiva com aparelhos auditivos electroacústicos. ${ }^{2-5}$ Várias pesquisas têm sustentado os efeitos deste método de reabilitação na diminuição do impacto da surdez, que se traduz na recuperação da capacidade auditiva, percepção dos sons e parâmetros segmentais da fala e, por conseguinte, no desenvolvimento da linguagem oral em crianças implantadas precocemente. ${ }^{1,6-10}$ O implante coclear, sobretudo quando realizado antes dos três anos de idade, permite que a criança esteja exposta aos sons durante o designado período crítico, no qual se

1. Serviço de Otorrinolaringologia. Centro Hospitalar e Universitário de Coimbra. Coimbra. Portugal.

2. Faculdade de Medicina. Universidade de Coimbra. Coimbra. Portugal.

3. Departamento de Eletrónica, Telecomunicações e Informática. Universidade de Aveiro. Aveiro. Portugal.

$\triangle$ Autor correspondente: Daniela Ramos. tfdanielaramos@hotmail.com

Recebido: 17 de Novembro de 2014 - Aceite: 26 de Março de 2015 | Copyright $\odot$ Ordem dos Médicos 2015 
desenvolvem as capacidades neurológicas que permitem uma melhor aprendizagem da linguagem e da fala. ${ }^{11,12} \mathrm{Um}$ número crescente de estudos sobre crianças utilizadoras de implante coclear documenta que elas podem adquirir vocabulário e as estruturas sintácticas necessárias para comunicar através da linguagem oral como as crianças normo-ouvintes. ${ }^{13-17}$

Alguns dos métodos utilizados para investigar os benefícios do implante coclear na linguagem das crianças com surdez neurosssensorial profunda implantadas, consistem na utilização de padrões de comparação que permitam confrontar o desempenho linguístico actual da criança com implante coclear com o seu desempenho linguístico obtido em fases anteriores. ${ }^{14,15,18}$ Outros relacionam a linguagem da criança utilizadora de implante coclear com a de outras crianças com surdez profunda não implantadas ${ }^{19-21,26}$ ou comparam o desempenho actual da criança com o desempenho dos seus pares normo-ouvintes, com a mesma idade auditiva ou cronológica. 1,22-26,28,29

$\mathrm{Na}$ análise do processo de desenvolvimento da linguagem são utilizados vários instrumentos de avaliação que permitem examinar o desempenho linguístico das crianças. ${ }^{27,30}$ Em Portugal, são poucos os estudos que se debruçaram sobre este tema, sendo escassa a informação sobre os resultados da avaliação das competências linguísticas das crianças utilizadoras de implante coclear, em idade escolar.

O objectivo deste estudo é identificar as capacidades linguísticas de crianças utilizadoras de implante coclear, com idades compreendidas entre os nove anos e um mês e os 10 anos, e compará-las com os dados normativos para a população portuguesa normo-ouvinte, com a mesma idade auditiva.

\section{MATERIAL E MÉTODOS}

O presente estudo teve aprovação da Comissão de Ética do Centro Hospitalar e Universitário de Coimbra (CHUC) para ser realizado.

Foram estudadas 18 crianças, de um universo de 58 crianças, com idade cronológica compreendida entre os nove anos e um mês e os 10 anos (média de idade cronológica: nove anos e seis meses) e idade auditiva variando entre os seis anos e um mês e os seis anos e 10 meses (média de idade auditiva: seis anos e cinco meses), com surdez neurossensorial bilateral profunda congénita, utilizadoras de implante coclear. Delas, oito eram do género feminino e 10 do género masculino. Todas realizaram cirurgia no Serviço de Otorrinolaringologia, na Unidade Funcional de Implantes Cocleares do Centro Hospitalar e Universitário de Coimbra. Nessas crianças, a idade média do diagnóstico de surdez foi de 23,37 meses. Depois de confirmada a surdez neurossensorial, foram colocados aparelhos auditivos convencionais, que foram usados durante um tempo médio de quatro meses. Nenhuma criança esteve submetida a outra forma de comunicação antes da implantação. A idade da cirurgia de implante coclear foi em média de 33,93 meses, variando entre os 21 e os 50 meses. Depois da acti- vação do processador da fala do implante coclear, um mês após cirurgia, todas as crianças iniciaram sessões diárias de terapia da fala. As mesmas, e de acordo com o protocolo utilizado no CHUC têm uma duração média de três meses, com um mínimo de quatro sessões semanais.

A seleção dos sujeitos foi realizada aleatoriamente, de acordo com a data da rotina de atendimento das crianças implantadas, previamente agendadas pelo serviço. Foram utilizadas as sessões de terapia da fala de reavaliação pós colocação de implante coclear, para aplicação do instrumento de recolha de dados.

Os dados foram recolhidos com o instrumento de avaliação - Grelha de Observação da Linguagem - nível escolar (GOL-E). ${ }^{31}$ Este instrumento avalia três estruturas linguísticas, semântica, morfossintaxe e fonologia, e faz parte do Protocolo de Avaliação de Crianças com Implantes Cocleares de Coimbra (PAC-IC). A GOL-E está aferida para crianças portuguesas em idade escolar primária, com idade cronológica, entre os cinco anos e sete meses e os 10 anos e zero meses. Cada estrutura linguística é avaliada por várias provas. A primeira prova da estrutura semântica é a definição de palavras, com a qual se pretende analisar a capacidade de descrever conceitos. A segunda prova, nomeação de classes, avalia o domínio do vocabulário em relação a termos superordenados. A terceira prova, opostos, avalia o conhecimento da palavra contrária. A estrutura morfossintática é avaliada com base em quatro provas. A primeira, reconhecimento de frases agramaticais, pretende avaliar a capacidade para fazer juízos gramaticais. A segunda prova, coordenação e subordinação de frases, avalia a capacidade de construção de frases complexas com coordenações e/ou subordinações a partir de frases simples. A terceira e quarta provas, ordem de palavras na frase e derivação de palavras avaliam a capacidade de ordenação das palavras para formar frases e o uso de regras morfológicas para criar palavras derivadas, respetivamente. Para a estrutura fonológica são usadas quatro provas. As duas primeiras, discriminação de palavras e discriminação de pseudo-palavras são provas que calculam a capacidade de discriminação auditiva. A identificação de palavras que rimam e a segmentação silábica são outras duas provas que fazem parte desta estrutura. A primeira visa avaliar a capacidade de identificar rimas e a segunda, a capacidade de segmentar palavras em sílabas. ${ }^{31}$

Para proceder à análise e comparação da pontuação das crianças utilizadoras de implante coclear com as crianças normo-ouvintes, foi utilizado como referência, a idade auditiva das crianças com implante coclear. A idade auditiva é o tempo decorrido entre a activação do processador da fala do implante coclear e a última avaliação linguística da criança utilizadora de implante coclear. As suas pontuações foram comparadas com os dados normativos (padrão) existentes na GOL-E ${ }^{31}$ para crianças normo-ouvintes da faixa etária dos 6;01-7;00 anos.

Para a análise estatística dos dados foi usado o One Sample $t$ test do programa SPSS 20 , com nível de significância mínimo de $p=0,05$. 


\section{RESULTADOS}

$\mathrm{Na}$ avaliação geral das três estruturas linguísticas estudadas, verificámos o seguinte:

$\mathrm{Na}$ estrutura semântica as crianças com implante coclear obtiveram uma pontuação média estatisticamente semelhante ao padrão $18,55 \pm 8,89$ vs. $19,20 \pm 4,85, p=$ 0,76 , alcançando uma percentagem de $96,3 \%$ em relação às capacidades linguísticas das normo-ouvintes.

A pontuação das crianças implantadas, na morfossintaxe por seu turno foi de $21,89 \pm 12,85$, relativamente menor do que o valor padrão para as crianças normo-ouvintes, $26,35 \pm 10,36$, significando $83,07 \%$ em relação ao padrão. A diferença, entretanto, não foi estatisticamente significativa, $p=0,159$.

A pontuação média obtida pelas crianças implantadas na fonologia, $29,44 \pm 8,4$, foi estatisticamente igual ao valor padrão, das crianças normo-ouvintes, 29,68 $\pm 5,90$, com $p$ $=0,91$, e representou $99,1 \%$ do valor médio das crianças normo-ouvintes. Estes dados estão apresentados na Fig. 1 .

$\mathrm{Na}$ análise das três provas que constituem a estrutura semântica, verificou-se que os resultados foram estatisticamente similares, em relação aos valores padrão, como é observável na Fig. 2. Na prova de definição de palavras, a pontuação das crianças implantadas foi de 7,1 $\pm 4,2$ vs. $8,13 \pm 2,20$ das normo-ouvintes, $p=0,29$. Na nomeação de classes, a pontuação média das crianças com implante coclear foi superior, embora estatisticamente semelhante ao valor padrão, $5,9 \pm 2,8$ vs. $5,15 \pm 1,96, p=0,28$. Na prova dos opostos a pontuação das crianças implantadas foi de $5,3 \pm 2,4$, sendo o padrão de $5,93 \pm 2,59, p=0,38$.

No que concerne à estrutura da morfossintaxe, a pontuação obtida na prova do reconhecimento de frases agramaticais, pelas crianças implantadas, quando comparada com o padrão dos normo-ouvintes, foi de 9,6 $\pm 6,0$ vs. 12,43 $\pm 4,73, p=0,065$. Nas provas de coordenação e subordinação de frases e ordenação de palavras em frase, os resultados foram muito semelhantes ao padrão, 3,8 $\pm 2,7$ vs. $3,53 \pm 3,11, p=0,63$ e $5,6 \pm 3,5$ vs. $5,73 \pm 3,69, p=$ 0,86 . Contrariamente, a capacidade de derivação de palavras foi significativamente baixa nas crianças utilizadoras de implante coclear. A pontuação média registada nessa prova foi de $2,83 \pm 2,81$ e os valores de referência de 4,65 $\pm 1,64, p=0,014$. Na Fig. 3 apresenta-se a comparação da pontuação nas provas da estrutura morfossintaxe das

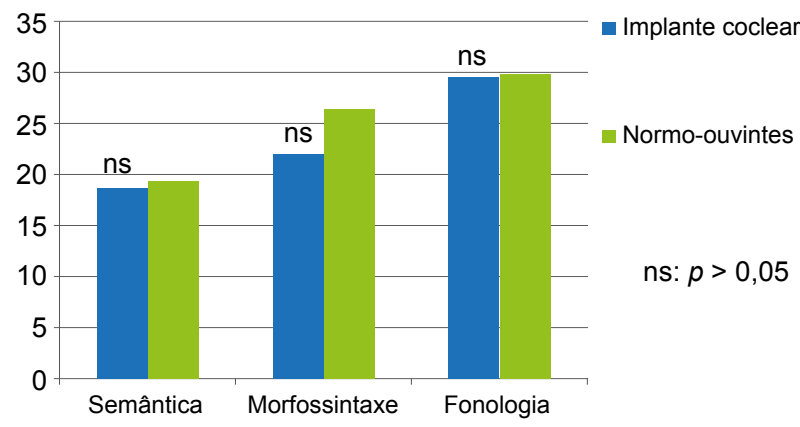

Figura 1 - Comparação da pontuação média das crianças com implante coclear com os valores das crianças normo-ouvintes, nas três estruturas que compõem a Grelha de Observação da Linguagem - nível escolar.

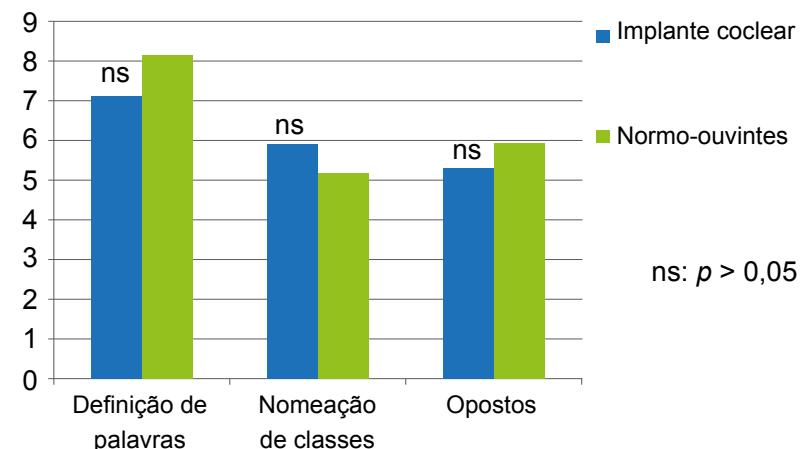

Figura 2 - Comparação da pontuação média das crianças com implante coclear com os valores médios das crianças normo-ouvintes, nas provas da estrutura da semântica da Grelha de Observação da Linguagem - nível escolar.

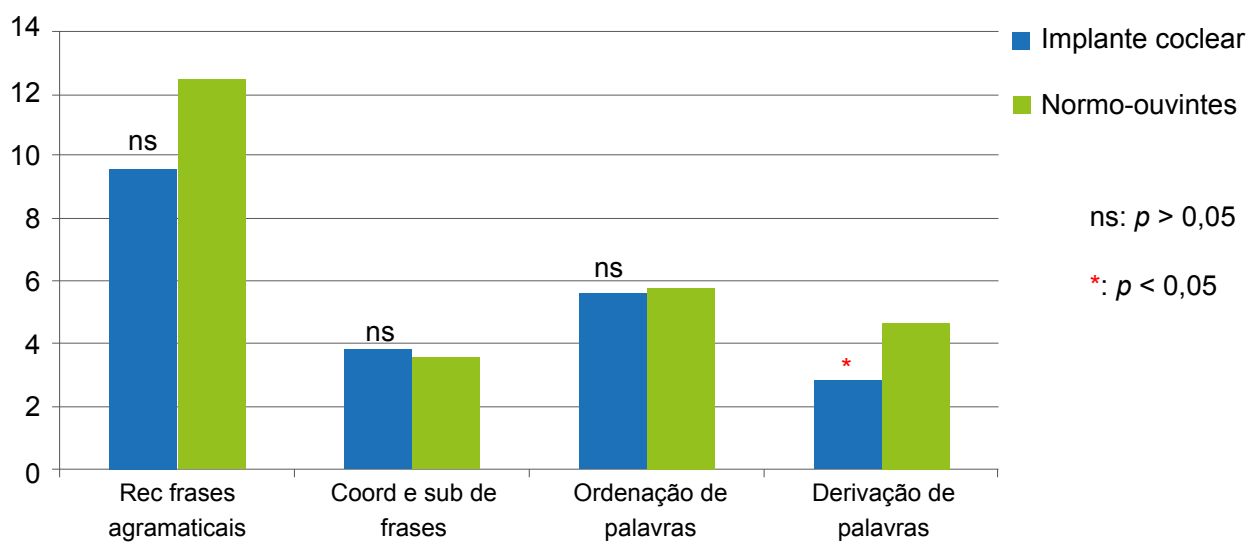

Figura 3 - Comparação da pontuação média das crianças com implante coclear com os valores médios das crianças normo-ouvintes, nas provas da estrutura da morfossintaxe da Grelha de Observação da Linguagem - nível escolar. 


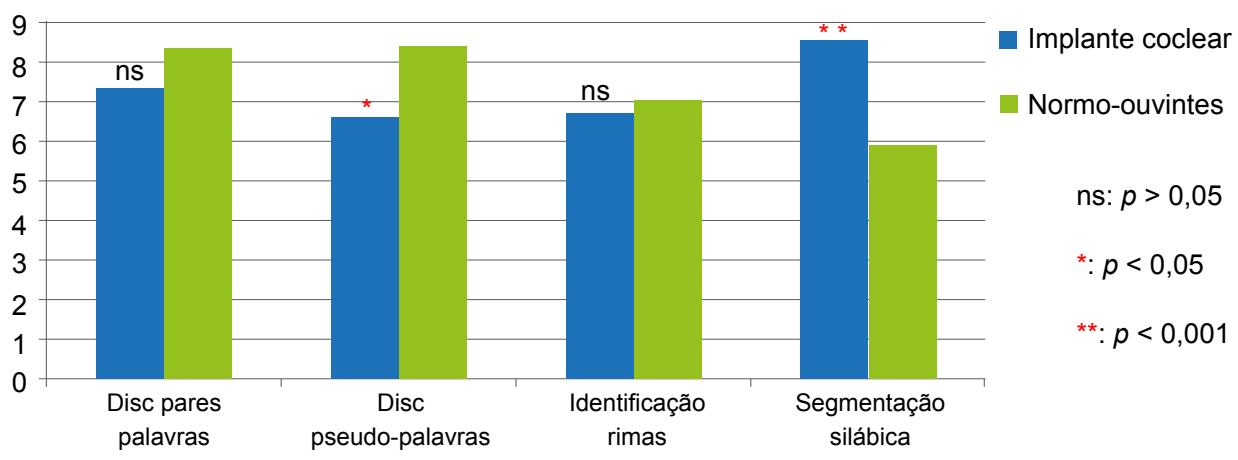

Figura 4 - Comparação da pontuação média das crianças com implante coclear com os valores médios das crianças normo-ouvintes, nas provas da estrutura da fonologia da Grelha de Observação da Linguagem - nível escolar

crianças com implante coclear estudadas com os valores padrão.

Nas provas da estrutura da fonologia, a pontuação na tarefa de discriminação de pares de palavras das crianças com implante coclear vs valor padrão, foi estatisticamente semelhante, $7,33 \pm 2,76$ vs. $8,35 \pm 2,55, p=0,137$, mas na discriminação de pseudo-palavras ficou significativamente aquém do valor de referência, $6,6 \pm 2,8$ vs. 8,37 $\pm 2,32, p=$ 0,023 . Na identificação de rimas, as crianças implantadas obtiveram uma prestação que se assemelhou estatisticamente ao valor das crianças normo-ouvintes, 6,7 $\pm 3,7$ vs. $7,05 \pm 2,30, p=0,712$. A prova da segmentação silábica foi onde se verificou uma prestação mais elevada das crianças implantadas, $8,56 \pm 1,6$ vs. 5,9 $\pm 1,58, p=0,001$. Os resultados das provas da fonologia estão plasmados na Fig. 4 .

\section{DISCUSSÃO}

O número, relativamente pequeno de crianças neste estudado constitui a sua maior limitação. Todavia os resultados obtidos permitem tecer algumas considerações que julgamos interessantes.

As crianças utilizadoras de implante coclear obtiveram pontuações que não diferiram estatisticamente, à das crianças normo-ouvintes nas três componentes linguísticas estudadas. Este facto, corrobora a ideia de que a utilização do implante coclear permite às crianças com surdez congénita profunda obterem ganhos importantes de linguagem verbal oral. A opção de utilizar um implante coclear, nas crianças com surdez congénita profunda, tem permitido ganhos no desenvolvimento da linguagem que os métodos anteriores provavelmente não terão possibilitado. A utilização de próteses auditivas convencionais, embora permita, nalgumas crianças, ganhos consideráveis de linguagem oral, o seu uso nas crianças com surdez neurossensorial profunda, apesar de as poder prover de benefícios na aquisição da linguagem oral, podem apresentar algumas limitações. Os estudos que compararam os ganhos em linguagem das crianças com próteses auditivas e as utilizadoras de implantes cocleares, registaram maiores competências linguísticas orais nas que tinham o implante coclear. ${ }^{15,32-34}$

A idade da implantação e o tempo durante o qual a criança utiliza o implante coclear são dois factores que influenciam de forma significativa as competências da linguagem oral adquiridas pelas crianças com surdez profunda. ${ }^{35-37} \mathrm{Nas}$ crianças alvo deste estudo, o facto de as mesmas terem a idade auditiva entre os seis anos e um mês e os seis anos e os 10 meses pode ter exercido influência importante nos resultados. O aumento do tempo de uso do implante coclear, e naturalmente da idade cronológica, condiciona maiores ganhos linguísticos nas crianças após a implantação, como é corroborado por vários autores. ${ }^{38,39}$

Os ganhos de linguagem em certas provas das estruturas estudadas, revelaram diferenças, algumas das quais muito significativas, em relação ao padrão. Na estrutura semântica, as pontuações obtidas pelas crianças implantadas, nas provas de definição de palavras, nomeação de classes e de opostos foram todas próximas aos de referência. Esta parece ser uma das estruturas em que os ganhos de linguagem reflectem, ainda que em parte, a intervenção e o seguimento da criança surda em consultas de terapia da fala e a sua exposição frequente, no seio familiar e escolar, a uma linguagem predominantemente oralista.

No que concerne aos componentes da estrutura da morfossintaxe, as crianças utilizadoras de implante coclear exibiram ganhos significativamente menores, em relação às normo-ouvintes, na derivação de palavras. Foi mesmo a mais baixa pontuação de todas as provas dos testes realizados. Este dado confirma a ideia, referida por alguns autores, deste constituir um domínio em que as crianças com implante coclear exibem maiores dificuldades. Boons et $\mathrm{al}^{40}$ verificaram estas dificuldades, em cerca de $50 \%$ das crianças com implante coclear que estudaram. Geers e Sedey ${ }^{41}$ verificaram fenómeno idêntico num estudo que envolveu 112 crianças, com idade igual ou superior a 10 anos, implantadas entre os dois e os cinco anos de idade cronológica. Todavia, nas outras tarefas da mesma estrutura, o reconhecimento de frases agramaticais e na coordenação e subordinação de frases, a pontuação média obtida pelas crianças implantadas não exibiu diferenças estatisticamente significativas. As crianças utilizadoras de implante coclear denotaram também, uma boa capacidade de ordenação de palavras para formar frases. As competências 
linguísticas da morfossintaxe reflectem em grande medida a aprendizagem escolar e a sua associação com o conjunto de conhecimentos absorvidos no dia-a-dia.

No domínio da fonologia, as crianças utilizadoras de implante coclear, apesar de exibirem uma pontuação média estatisticamente semelhante ao normal, a pontuação obtida, particularmente, na tarefa de discriminação de pseudo-palavras apontou para uma fraca prestação. Este facto está de acordo com a observação de Bouton, Colé e Serniclaes, que ao estudarem 25 crianças com implante coclear verificaram ser este um domínio em que as crianças com IC também apresentaram maior dificuldade. ${ }^{42}$ Ao contrário, na segmentação silábica, as crianças utilizadoras de implante coclear obtiveram resultados significativamente acima dos valores padrão. Os apoios da educação especial no âmbito escolar associado ao seguimento em terapia da fala têm grande relevância neste domínio, ${ }^{43,44}$ pelo que esta deve ser mais uma das tarefas em que, mercê destas intervenções, os ganhos se tornaram francamente evidentes.

Os dados obtidos neste estudo demonstraram que a utilização dos implantes cocleares em crianças com surdez severa a profunda constitui um importante meio de promoção de ganhos significativos na aprendizagem e utilização da linguagem verbal oral, como afirmaram Niparko et $a^{18}$ e Ostojić et $a^{28}$ nos seus estudos. Nalguns aspectos da linguagem, nomeadamente na discriminação de pseudo-palavras e na derivação de palavras o progresso pode ser mais lento, nesses domínios, nas fases precoces da idade auditiva e ficar aquém do desejável. ${ }^{42}$ Esta constatação impõe uma reflexão sobre as suas razões, que fundamente a busca de soluções para o progressivo aprimoramento da linguagem nas crianças com implante coclear. A solução pode passar por uma maior dedicação a estes aspectos pelos terapeutas da fala em conjugação com a intervenção escolar dos professores, que devem ser avisados da necessidade de dar uma atenção especial a estes aspectos da linguagem destas crianças. De qualquer forma, como referem Martini et al ${ }^{11}$ a utilização deste elemento facilitador do desenvolvimento da linguagem em crianças com surdez deve ser incentivado pois tem provado os seus benefícios para essas crianças, cuja deficiência pode colocar em risco a sua integração e prestação sociais e desenvolvimento pessoal.

\section{REFERÊNCIAS}

1. Fortunato $\mathrm{C}$, Bevilacqua $\mathrm{M}$, Costa $\mathrm{MD}$. Análise comparativa da linguagem oral de crianças ouvintes e surdas usuárias de implante coclear. Revista CEFAC. 2009;11:662-72.

2. Meinzen-Derr J, Wiley S, Grether S, Choo, D. Children with cochlear implants and developmental disabilities: A language skills study with developmentally matched hearing peers. Res Dev Disabil. 2011;32:75767.

3. Mukari S, Ling L, Ghani H. Educational performance of pediatric cochlear implant recipients in mainstream classes. Int J Pediatr Otorhinolaryngol. 2007;71:231-40.

4. Semenov YR, Yeh ST, Seshamani M, Wang NY, Tobey EA, Eisenberg LS, et al. Age-dependent cost-utility of pediatric cochlear implantation. Ear Hear. 2013;34:402-12.

5. Kim LS, Jeong SW, Lee YM, Kim JS. Cochlear implantation in children. Auris Nasus Larynx. 2010;37:6-17.
Consideramos que estudos com um maior número da amostra poderão permitir uma melhor avaliação dos parâmetros estudados.

\section{CONCLUSÕES}

As crianças com implante coclear estudadas, obtiveram genericamente bom desempenho nas provas das estruturas da fonologia, morfo-sintaxe e da semântica, com valores similares ao padrão para as crianças normo-ouvintes. Os ganhos de linguagem das crianças com implante coclear revelaram-se significativamente menores na prova de derivação de palavras da morfossintaxe e na discriminação de pseudo-palavras da fonologia. Este estudo, apesar do pequeno número de indivíduos que abrangeu, demonstrou, como se refere na literatura, que o implante coclear é um método de reabilitação auditiva eficaz na diminuição do impacto da surdez neurossensorial severa a profunda e na promoção do desenvolvimento da linguagem verbal oral nas crianças com surdez congénita bilateral profunda.

\section{AGRADECIMENTOS}

Os autores agradecem às terapeutas da fala Marisa Alves e Helena Alves pelo valioso contributo na recolha dos dados, a todos os médicos e técnicos que participaram no ato de implantação coclear e aos pais/cuidadores e crianças envolvidas no estudo.

\section{PROTECÇÃO DE PESSOAS E ANIMAIS / CONFIDEN- CIALIDADE DOS DADOS}

O presente estudo teve aprovação da Comissão de Ética do Centro Hospitalar e Universitário de Coimbra para ser realizado. Os autores declaram ter seguido os protocolos do seu centro de trabalho acerca da publicação dos dados de doentes.

\section{CONFLITO DE INTERESSES}

Os autores declaram não ter nenhum conflito de interesses relativamente ao presente artigo.

\section{FONTES DE FINANCIAMENTO}

Não existiram fontes externas de financiamento para a realização deste artigo.

6. Anderson I, Weichbold V, D'Haese P, Szuchnik J, Quevedo M, Martin $\mathrm{J}$, et al. Cochlear implantation in children under the age of two-what do the outcomes show us? Int J Pediatr Otorhinolaryngol. 2004;68:425-31.

7. Moret $\mathrm{M}$, Bevilacqua $\mathrm{M}$, Costa $\mathrm{O}$. Implante coclear: audição e linguagem em crianças deficientes auditivas pré-linguais. Pró-Fono Rev Atualização Cient. 2007;19:295-304.

8. Wolfgang K, Jafar H, Brigitte E, Wolf D. Speech perception performance in prelingually deaf children with cochlear implants. Acta Otolaryngol. 2000;120:209-13.

9. Polat B, Başaran B, Kara HC, Ataş A, Süoğlu Y. The impact of social and demographic features on comprehensive receptive and expressive performance in cochlear implant patients. Kulak Burun Bogaz Ihtis Derg. 2013;23:90-5

10. Mlynski R, Plontke S. Cochlear implants in children and adolescents. HNO. 2013:61:388-98. 
11. Martini A, Bovo R, Trevisi P, Forli F, Berrettini S. Cochlear implant in children: rational, indications and cost/efficacy. Minerva Pediatr. 2013;65:325-39.

12. Fitzpatrick $E$, Johnson $E$, Durieux-Smith A. Exploring factors that affect the age of cochlear implantation in children. International J Pediatr Otorhinolaryngol. 2011;75:1082-7.

13. Ouellet $\mathrm{C}$, Le Normand $\mathrm{M}$, Cohen $\mathrm{H}$. Language evolution in children with cochlear implants. Brain Cogn. 2001;46:231-5.

14. Chilosi AM, Comparini A, Scusa MF, Orazini L, Forli F, Cipriani P, et al. A longitudinal study of lexical and grammar development in deaf Italian children provided with early cochlear implantation. Ear Hearing 2013;34:e28-37.

15. Svirsky MA, Robbins AM, Kirk KH, Pisoni DB, Miyamoto RT. Language development in profoundly deaf children with cochlear implants. Psychological Sci. 2000;11:153-8.

16. Ge'raeda JM, Deggouj N, Hupina C, Buissonb AL, Monteyne V, Lavis C, et al. Evolution of communication abilities after cochlear implantation in prelingually deaf children. Int J Pediatr Otorhinolaryngol. 2010;74:642-8.

17. Toe DM, Paatsch LE. The conversational skills of school-aged children with cochlear implants. Cochlear Implants Int. 2013;14:67-79.

18. Niparko J, Tobey E, Thal D, Eisenberg L, Wang NY, Quittner A, et al. Spoken language development in children following cochlear implantation. JAMA. 2010;303:1498-506.

19. Fitzpatrick EM, Olds J, Gaboury I, McCrae R, Schramm D, DurieuxSmith A. Comparison of outcomes in children with hearing aids and cochlear implants. Cochlear Implants Int. 2012;13:5-15.

20. Yoshinaga-Itano C, Baca RL, Sedey AL. Describing the trajectory of language development in the presence of severe-to-profound hearing loss: a closer look at children with cochlear implants versus hearing aids. Otol Neurotol. 2010;31:1268-74.

21. Most T, Shina August E, Meilijson S. Pragmaticabilities of children with hearing loss using cochlear implants or hearing AIDS compared to hearing children. J Deaf Stud Deaf Educ. 2010;15:422-37.

22. Tsiakpini L, Weichbold V, Coninx F. Manual do Questionário AuditivoLittle Ears. MED-EL. 2003.

23. Nikolopoulos TP, Dyar D, Archbold S, O'Donoghue GM. Development of spoken language grammar following cochlear implantation in prelingually deaf children. Arch Otolaryngol Head Neck Surg. 2004;130:629-33.

24. May-Mederake B. Early intervention and assessment of speech and language development in young children with cochlear implants. Int J Pediatr Otorhinolaryngol. 2012;76:939-46.

25. Robbins AM, Bollard PM, Green J. Language development in children implanted with the CLARION cochlear implant. Ann Otol Rhinol Laryngol. 1999;177:113-8.

26. Iwasaki S, Nishio S, Moteki H, Takumi Y, Fukushima K, Kasai N, et al. Language development in Japanese children who receive cochlear implant and/or hearing aid. Int J Pediatr Otorhinolaryngol. 2012;76:433-

27. Sugishita S, Fukushima K, Kasai N, Konishi T, Omori K, Taguchi T, et al Language development, interpersonal communication, and academic achievement among Japanese children as assessed by the ALADJIN. Ann Otol Rhinol Laryngol. 2012;202:35-39.
28. Ostojić S, Djoković S, Dimić N, Mikić B. Cochlear implant-speech and language development in deaf and hard of hearing children following implantation. Vojnosanitetski Pregled. 2011;68:349-52.

29. Leigh J, Dettman S, Dowell R, Briggs R. Communication development in children who receive a cochlear implant by 12 months of age. Otol Neurotol. 2013;34:443-50.

30. Silva MP, Comerlatto Junior AA, Bevilacqua MC, Lopes-Herrera SA. Instruments to assess the oral language of children fitted with a cochlear implant: a systematic review. J Appl Oral Sci. 2011;19:549-53.

31. Sua Kay E, Santos M, Ferreira A, Graça D, Calado A. Grelha de observação da linguagem - nível escolar. Lisboa: Alcoitão: Escola Superior de Sáude do Alcoitão; 2003.

32. Tait M, Lutman ME. Comparison of early communicative behavior in young children with cochlear implants and with hearing aids. Ear Hear 1994;15:352-61.

33. Baldassari C, Schmidt C, Schubert C, Srinivasan P, Dodson K, Sismanis A. Receptive language outcomes in children after cochlear implantation. Otolaryngology-Head and Neck Surgery. 2009;140:114-9.

34. Geers A, Moog J. Spoken language results: vocabulary, syntax, and communication. Volta Rev. 1994;96:131-50.

35. Lee $\mathrm{Y}$, Yim D, Sim H. Phonological processing skills and its relevance to receptive vocabulary development in children with early cochlear implantation. Int J Pediatr Otorhinolaryngol. 2012;76:1755-60.

36. Schramm B, Bohnert A, Keilmann A. Auditory, speech and language development in young children with cochlear implants compared with children with normal hearing. Int J Pediatr Otorhinolaryngol. 2010;74:812-9

37. Fitzpatrick EM, Johnson E, Durieux-Smith A. Exploring factors that affect the age of cochlear implantation in children. Int $\mathrm{J}$ Pediatr Otorhinolaryngol. 2011;75:1082-7.

38. Colletti V, Carner M, Miorelli V, Guida M, Colletti L, Fiorino F. Cochlear implantation at under 12 months: Report on 10 patients. Laryngoscope. 2005;115:445-9.

39. Manrique M, Cervera-Paz F, Huarte A, Molina M. Advantages of cochlear implantation in prelingual deaf children before 2 years of age when compared with later implantation. Laryngoscope. 2004;114:1462-9.

40. Boons T, De Raeve L, Langereis M, Peeraer L, Wouters J, Van Wieringen A. Expressive vocabulary, morphology, syntax and narrative skills in profoundly deaf children after early cochlear implantation. Res Dev Disabil. 2013;34:2008-22.

41. Geers A, Sedey A. Language and verbal reasoning skills in adolescents with 10 or more years of cochlear implant experience. Ear Hearing. 2011:32:39S-48

42. Bouton $S$, Colé $P$, Serniclaes $W$. The influence of lexical knowledge on phoneme discrimination in deaf children with cochlear implants. Speech Communication. 2012;54:189-98.

43. Stuchi RF, Nascimento LT, Bevilacqua MC, Brito Neto RV. Linguagem oral de crianças com cinco anos de uso do implante coclear. Pró-Fono Rev Atualização Científica. 2007;19:167-76.

44. Soman U, Kan D, Tharpe A. Rehabilitation and educational considerations for children with cochlear implants. Otolaryngol Clin N Am. 2012:45:141-53. 


\section{O Impacto do Implante Coclear na Linguagem Oral das Crianças com Surdez Congénita}

Acta Med Port 2015:28:442-447

Publicado pela Acta Médica Portuguesa, a Revista Científica da Ordem dos Médicos

Av. Almirante Gago Coutinho, 151

1749-084 Lisboa, Portugal.

Tel: +351218428 215

E-mail: submissao@actamedicaportuguesa.com

www.actamedicaportuguesa.com

ISSN:0870-399X | e-ISSN: 1646-0758

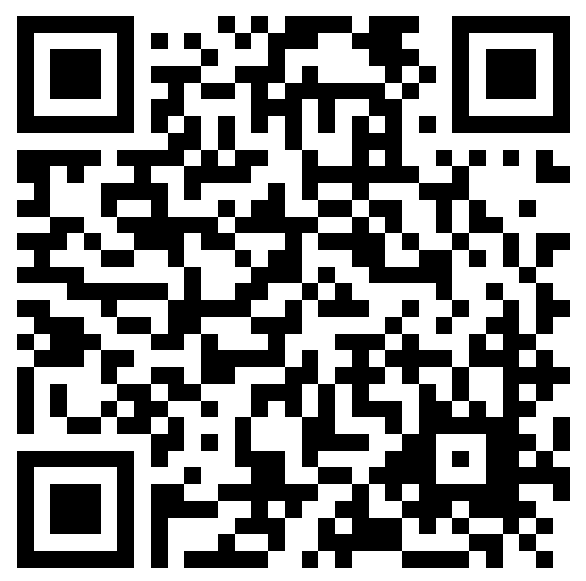

